\title{
Comparative analysis of miRNA expression during the development of insects of different metamorphosis modes and germ- band types
}

\author{
Guillem Ylla, Maria-Dolors Piulachs ${ }^{*}$ and Xavier Belles ${ }^{*}$ (1)
}

\begin{abstract}
Background: Do miRNAs contribute to specify the germ-band type and the body structure in the insect embryo? Our goal was to address that issue by studying the changes in miRNA expression along the ontogeny of the German cockroach Blattella germanica, which is a short germ-band and hemimetabolan species.

Results: We sequenced small RNA libraries representing 11 developmental stages of B. germanica ontogeny (with especial emphasis on embryogenesis) and the changes in miRNA expression were examined. Data were compared with equivalent data for two long germ-band holometabolan species Drosophila melanogaster and Drosophila virilis, and the short germ-band holometabolan species Tribolium castaneum. The identification of B. germanica embryo small RNA sequences unveiled miRNAs not detected in previous studies, such as those of the MIR-309 family and 54 novel miRNAs. Four main waves of miRNA expression were recognized (with most miRNA changes occurring during the embryonic stages): the first from day 0 to day 1 of embryogenesis, the second during mid-embryogenesis (days 0-6), the third (with an acute expression peak) on day 2 of embryonic development, and the fourth during post-embryonic development. The second wave defined the boundaries of maternal-to-zygotic transition, with maternal mRNAs being cleared, presumably by Mir-309 and associated scavenger miRNAs.
\end{abstract}

Conclusion: miRNAs follow well-defined patterns of expression over hemimetabolan ontogeny, patterns that are more diverse during embryonic development than during the nymphal stages. The results suggest that miRNAs play important roles in the developmental transitions between the embryonic stages of development (starting with maternal loading), during which they might influence the germ-band type and metamorphosis mode.

Keywords: miRNAs, Embryogenesis, Maternal-to-zygotic transition, Germ-band, Metamorphosis, Blattella, Tribolium, Drosophila

\section{Background}

Do embryonic miRNAs influence the germ-band type and metamorphosis mode of insects? Long germ-band insects, such as the fruit fly Drosophila melanogaster, form most of their segments simultaneously at the blastoderm stage, i.e., before gastrulation. In contrast, short germ-band species start gastrulation with just a few segments, and then progressively add new ones from

\footnotetext{
*Correspondence: mdolors.piulachs@ibe.upf-csic.es; xavier.belles@ibe.upfcsic.es

Institute of Evolutionary Biology, CSIC-Universitat Pompeu Fabra, Passeig Marítim 37, 08003 Barcelona, Spain
}

(c) The Author(s). 2017 Open Access This article is distributed under the terms of the Creative Commons Attribution 4.0 International License (http://creativecommons.org/licenses/by/4.0/ which permits unrestricted use, distribution, and reproduction in any medium, provided you give appropriate credit to the original author(s) and the source, provide a link to the Creative Commons license, and indicate if changes were made. The Creative Commons Public Domain Dedication waiver (http://creativecommons.org/publicdomain/zero/1.0/) applies to the data made available in this article, unless otherwise stated.

an undifferentiated growth zone situated at the posterior end of the embryo [1]. Short germ-band development is typical of basal insects such as locusts and cockroaches, whereas more derived species such as those of the genus Drosophila predominantly undergo long germ-band development [2]. The differences between these two developmental extremes are paralleled by the differential expressions of patterning genes [3, 4]. In general, the participation of miRNAs in gene-regulatory networks is crucial, increasing the precision of expression and reducing noise [5-9]. It is therefore likely that miRNAs influence the mechanisms that specify the germ-band 
type and body structure, which is related to the metamorphosis mode, in insect embryos.

We examined the changes in miRNA expression over the ontogeny of the German cockroach Blattella germanica, using 11 small RNA libraries prepared and sequenced in our laboratory, representing developmentally important stages. The basic details of the miRNA complement of $B$. germanica, a short germ-band, hemimetabolan species, are known $[10,11]$, as are a number of miRNA functions, especially in postembryonic development [12-15]. In this work, the small RNA libraries of $B$. germanica early embryo were compared with those previously published of two long germ-band holometabolan species, the flies D. melanogaster and Drosophila virilis, and the red flour beetle Tribolium castaneum, a short germ-band holometabolan species $[16,17]$. The distinction between the hemimetabolan and holometabolan modes of metamorphosis is important: hemimetabolan species develop the basic adult body structure during embryogenesis, whereas holometabolans delay this until the pupal stage $[18,19]$. miRNA commonalities between B. germanica and T. castaneum, as opposed to those of the two Drosophila species studied, were assumed to be associated with the germ-band type, whereas those of T. castaneum and the Drosophila species, as opposed to $B$. germanica, were assumed to be associated with the mode of metamorphosis followed.

\section{Results}

Novel miRNAs in the cockroach embryo

B. germanica embryogenesis was completed in 18 days at the set temperature of $29{ }^{\circ} \mathrm{C}$. Nymph development in colony reared at this temperature lasted between 22 and 24 days and comprised six instars. Eleven stages covering essential developmental moments and characteristic hormonal contexts (Table 1) were chosen for examination. Two libraries of small RNAs were prepared for each examined stage and sequenced using Illumina NextSeq technology. This provided 292 million paired end reads, with each library having 9.2-17 million read pairs. After removing adapters, filtering out low quality reads, and merging read pairs, 148,674,256 million reads were mapped to the $B$. germanica genome and used in analyses (Additional file 1: Table S1).

Putative B. germanica miRNAs were then predicted using the mirDeep2 algorithm. The predictions included the 88 conserved insect miRNAs and 11 miRNAs previously discovered in B. germanica [10, 11], plus 264 novel miRNA candidates. After filtering the 264 candidates adhering to the biogenesis criteria [11, 20], 67 bona-fide $B$. germanica miRNA genes were obtained (Additional file 2: Table S2). Four of these belonged to the MIR-309 family (Fig. 1a), which is typical of insects [11] and that in $D$. melanogaster is involved in the clearance of maternally

Table 1 Biological data corresponding to the 11 small RNA libraries used in the present work

\begin{tabular}{|c|c|c|c|c|c|}
\hline Library & Developmental period & Age (AO: after oviposition) & $\begin{array}{l}\text { Percentage of embryo } \\
\text { development (ED) }\end{array}$ & $\begin{array}{l}\text { Embryo development and } \\
\text { Tanaka (1976) stage }\end{array}$ & Hormonal context \\
\hline NFE & Non-fertilized egg & $\begin{array}{l}\text { Day } 8 \text { of the first } \\
\text { gonadotrophic cycle } \\
\text { (in preoviposition) }\end{array}$ & - & - & $\begin{array}{l}\text { Not determined within the } \\
\text { egg. High levels of } 20 \mathrm{E} \text { and } \\
\mathrm{JH} \text { in the surrounding } \\
\text { haemolymph. }\end{array}$ \\
\hline EDO & Embryo & $\begin{array}{l}8 \mathrm{~h} \mathrm{AO} \text {, when the ootheca } \\
\text { is still vertical }\end{array}$ & $2 \%$ & Only yolk granules observed. & $\begin{array}{l}\text { No detectable levels of } 20 E \\
\text { and } \mathrm{JH}\end{array}$ \\
\hline ED1 & Embryo & $24 \mathrm{~h} \mathrm{AO}$ & $6 \%$ & $\begin{array}{l}\text { Energids at low density spread } \\
\text { among the yolk granules. Tanaka } \\
\text { stage } 1\end{array}$ & $\begin{array}{l}\text { No detectable levels of } 20 \mathrm{E} \\
\text { and } \mathrm{JH}\end{array}$ \\
\hline ED2 & Embryo & $48 \mathrm{~h} \mathrm{AO}$ & $12 \%$ & $\begin{array}{l}\text { Abundant energids, germ band } \\
\text { anlage well delimited, slightly } \\
\text { expanded at both sides. Tanaka } \\
\text { stage } 2\end{array}$ & $\begin{array}{l}\text { Burst of } 20 \mathrm{E} \text { inferred from the } \\
\text { expression of HR3 (a 20E- } \\
\text { dependent gene). No detectable } \\
\mathrm{JH}\end{array}$ \\
\hline ED6 & Embryo & $144 \mathrm{~h} \mathrm{AO}$ & $33 \%$ & $\begin{array}{l}\text { Pleuropodia well apparent, legs } \\
\text { segmented, caudal space arises. } \\
\text { Tanaka stage } 8\end{array}$ & $\begin{array}{l}\text { Peak of 20E. Very low levels } \\
\text { of JH }\end{array}$ \\
\hline ED13 & Embryo & $312 \mathrm{~h} \mathrm{AO}$ & $72 \%$ & $\begin{array}{l}\text { Eyes well colored, antennae and } \\
\text { legs reaching the } 5 \text { th abdominal } \\
\text { segment. Tanaka stage } 15\end{array}$ & Peak of 20E. High levels of $\mathrm{JH}$ \\
\hline N1 & 1st nymphal instar & $1-2$ days old & - & - & High levels of $20 \mathrm{E}$ and $\mathrm{JH}$ \\
\hline N3 & 3rd nymphal instar & 2-4 days old & - & - & High levels of $20 \mathrm{E}$ and $\mathrm{JH}$ \\
\hline N5 & 5th nymphal instar & 3-5 days old & - & - & High levels of $20 \mathrm{E}$ and $\mathrm{JH}$ \\
\hline N6 & 6th nymphal instar & 5-7 days old & - & - & High levels of $20 \mathrm{E}, \mathrm{no} \mathrm{JH}$ \\
\hline Adult & Adult (female) & 5 days old & - & - & $\begin{array}{l}\text { Low (ovarian) levels of } 20 \mathrm{E} \\
\text { and high JH levels }\end{array}$ \\
\hline
\end{tabular}



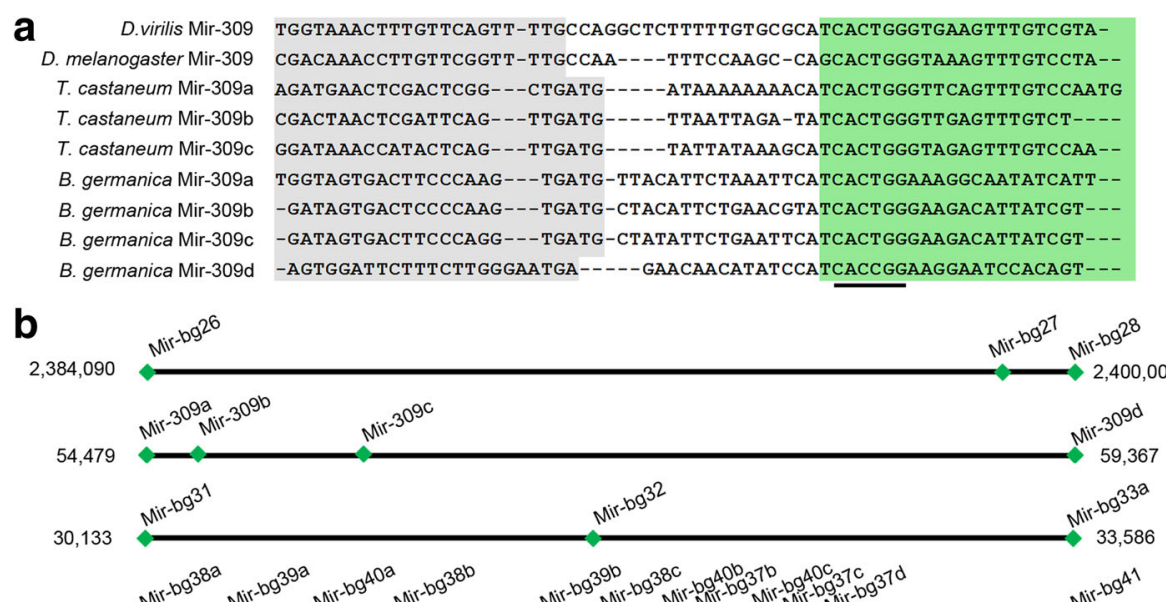

$358,436 \stackrel{N}{\longrightarrow} 382689$
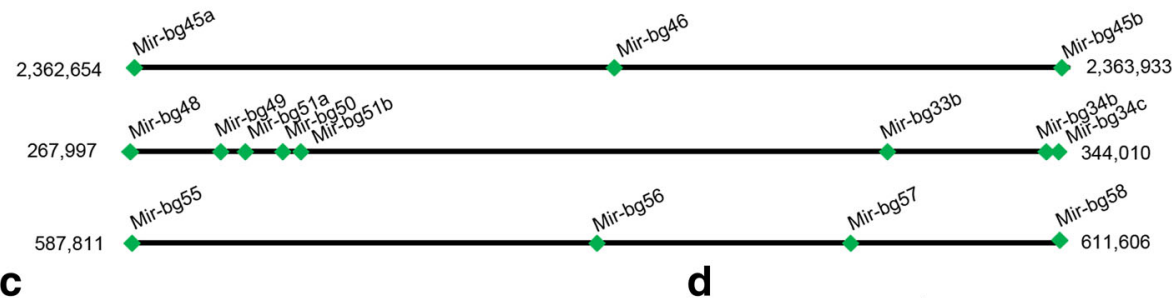

C
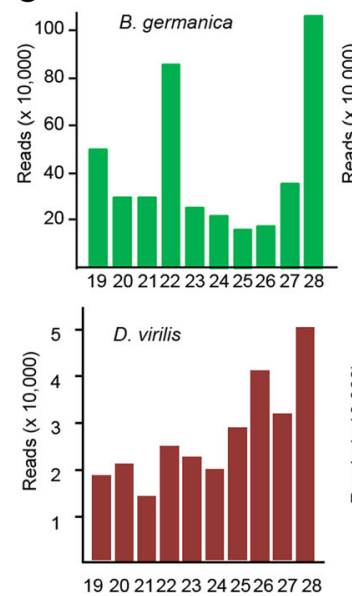
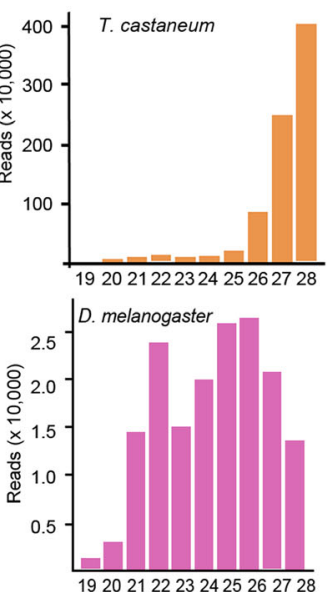

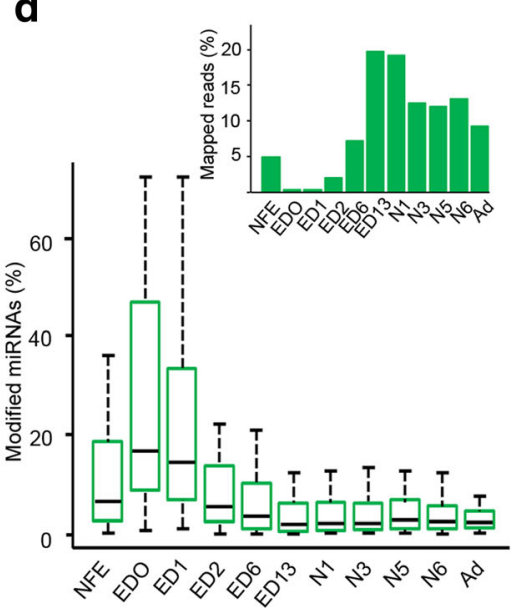

Fig. 1 B. germanica miRNAs. a Precursor sequences of the MIR-309 miRNAs in B. germanica, T. castaneum, D. melanogaster and D. virilis. Highlighted in grey are the sequences corresponding to star miRNAs, and in green those corresponding to mature miRNAs. The conserved seed sequence is underlined. b Genomic clusters of newly identified B. germanica miRNAs containing more than 3 miRNA genes. c Read size distribution in the NFE small RNA libraries of the studied species; read length (abscissae) is expressed in number of nucleotides. d Frequency of miRNAs with 3-end modified reads in the developmental stages studied. The inset shows the relative abundance of miRNA reads per library. The abbreviations for the stages are explained in Table 1

loaded mRNAs [21]. As no known orthologs of the other 63 miRNA genes were found, they were considered as newly discovered. Six of these genes contained between two and three identical, mature miRNAs sequences. Thus, 54 different novel mature miRNAs, grouped into 48 miRNA families, were finally identified as novel in $B$. germanica (Additional file 2: Table S2). These $54 \mathrm{miR}-$ NAs, plus the 11 previously reported [11], represent the full repertoire miRNAs only found in B. germanica. As seen for other B. germanica miRNAs [11], most of those reported herein for the first time grouped into genomic clusters, e.g., into the MIR-309 family (Fig. 1b). Moreover, 43 of the 65 miRNA genes now discovered in $B$. germanica grouped into nine additional clusters spanning 4-70 kb, and included 2-12 usually paralogous miRNA genes per cluster (Fig. 1b).

With respect to maternal miRNAs (non-fertilized eggs, NFE, library), the read length distribution (Fig. 1c) showed two prominent peaks, one at 22 nts corresponding to miRNAs, and the other at 28 nts that might 
correspond to Piwi-interacting RNAs (piRNAs) [16, 22]. Comparison of the NFE libraries of $T$. castaneum, $D$. melanogaster and $D$. virilis $[16,17]$ revealed the proportion of miRNAs and piRNAs to be different in T. castaneum (Fig. 1c), with the latter very much more abundant [17]. Interestingly, the B. germanica miRNAs in embryo day (ED) 0 and ED1 showed a higher frequency $(15-26 \%)$ of reads with extra nucleotides at the 3 '-end compared to other embryo or post-embryo stages (7-11\%). Most of these 3 '-end modifications were adenylations (approximately 50\%) and uracylations (25\%) (Fig. 1d). The peak of 3 '-end modifications at ED0-ED1 coincide with the minimum relative number of miRNA reads (Fig. 1d, inset).

\section{Changes in miRNA expression over cockroach ontogeny}

Most of the newly discovered miRNAs predominated in the embryo, especially in the early stages, from ED0 to ED2 (Fig. 2a, Additional file 3: Table S3). In contrast, conserved miRNAs tended to be expressed over a longer period of time (Fig. 2b, Additional file 3: Table S3). Therefore, the new and predominantly embryonic miRNAs showed a generally higher coefficient of variation in their expression over development (Fig. 2c, Additional file 4: Figure S1). The expression patterns of the 157 B. germanica miRNAs were very diverse (Fig. 2), but coexpression networks recognized through the use of Spearman correlation analysis allowed this diversity to be collapsed into four main co-expression modules (CoMod) (Fig. 3). CoMod-A grouped miRNAs generally expressed during embryonic development. In fact, CoMod-A can be subdivided into two sub-modules (Fig. 3): CoMod-A1, which groups miRNAs predominantly expressed during ED0-ED1, and CoMod-A2, which groups those mostly expressed between ED0 and ED6. CoMod-B is characterized by acute expression during ED2, thereafter decreasing in intensity. Finally, CoMod-C grouped miRNAs expressed during post-embryonic development. The respective metagene expression patterns of these four CoMods are shown in Fig. 3.

Other, peculiar miRNA expression patterns were also seen. For example, Mir-bg5 was (strongly) expressed in very early embryos and in adult females (Fig. 4a). The comparison of Mir-252b and Mir-252a is interesting since both share the same seed sequence (although the corresponding genes are separated by some $40 \mathrm{~kb}$ in the genome). However, Mir-252b showed an expression peak during ED6 and a dramatic reduction in expression in ED13, while Mir-252a expression dramatically increased from ED13 onward (Fig. 4a). The expression of Mir-309 followed a CoMod-B pattern, showing an acute peak in ED2 (at 11\% of development) (Fig. 4b). This is compatible with a possible role in the clearance of maternally loaded mRNAs, as seen in D. melanogaster [21]. The expression pattern of Mir-309 in D. virilis showed a peak at $13-20 \%$ of development (Fig. 4b), similar to that seen for B. germanica, whereas in T. castaneum, Mir-309 expression increased at $6-11 \%$ of development and then remained more or less stable until the end of embryogenesis (Fig. 4b).

Evidence of differential expression between stagelibraries was also sought. The transition between NFE and ED0 involved more changes than between ED1ED13. The most conservative transitions were those between ED0 and ED1 (during which only one miRNA was downregulated), and between N5 and N6 (during which no changes were observed) (Fig. 4c, Additional file 5: Figure S2). Principal component analysis (PCA) of the expression data of all miRNAs in all libraries (Fig. 4d) highlighted the contrast between the compact group composed by nymphal and adult stages and that formed by the embryonic stages.

\section{Maternal and very early embryonic miRNAs in Blattella, Tribolium and Drosophila}

To study the possible role of miRNAs in the definition of the germ-band type, the B. germanica NFE library (Table 1) was compared with the equivalent nonfertilized egg libraries of $T$. castaneum, $D$. melanogaster and $D$. virilis $[16,17]$. Clustering analysis of the expression of orthologous miRNA families in the four species (Fig. 5a) separated the long germ-band species (D. melanogaster and $D$. virilis) from the two short germ-band species (T. castaneum and B. germanica). PCA (Fig. 5b) showed this grouping to be mostly driven by the MIR-276, MIR-279 and MIR-92 families. Though not highlighted by PCA, the miRNAs from the let-7 cluster were significantly expressed in B. germanica and T. castaneum NFE, but not in the Drosophila species studied. Further, the MIR-bg5 family was highly expressed in B. germanica (the fifth most abundant miRNA family), but not in T. castaneum or the Drosophila species.

Comparisons were also made between $B$. germanica ED0 ( $8 \mathrm{~h}, 1.9 \%$ of development) library (Table 1 ) and the equivalent libraries for the first hours of embryonic development in $T$. castaneum $(0-5 \mathrm{~h}, 0-3.5 \%$ of development) and $D$. virilis (0-2 h, 0-6.7\% development) [16, 17]. In all three, the germ-band began to form at this time. The miRNA expression profiles were notably similar for the three species; indeed, clustering analysis hardly separated the long germ-band species $(D$. virilis) from the two short germ-band species ( $T$. castaneum and B. germanica) (Fig. 6a). PCA showed the main drivers of this grouping to again be MIR-279 (for $B$. germanica and T. castaneum) and MIR-92 (for D. virilis) (Fig. 6b). 


\section{a}

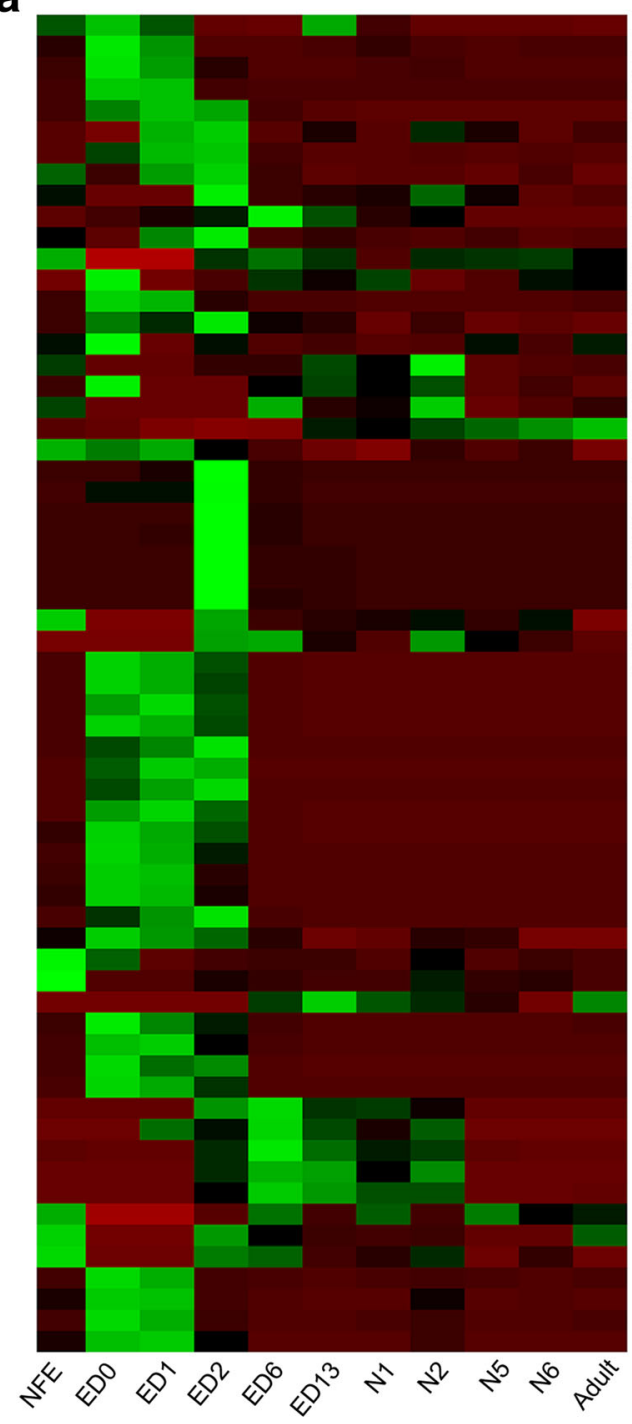

C

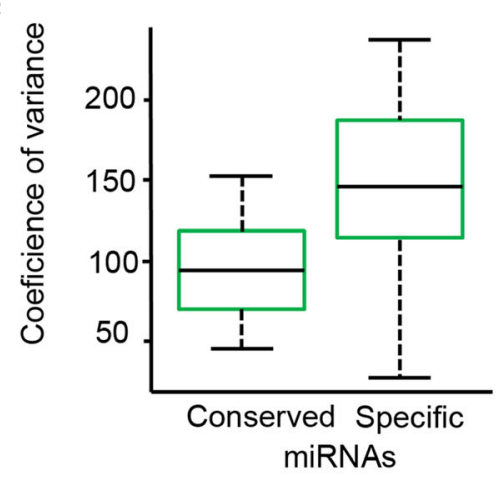

b

Mir-bg10

Mir-bg11

Mir-bg12

Mir-bg13

Mir-bg14

Mir-bg15

Mir-bg16

Mir-bg17
Mir-bg18

Mir-bg19

Mir-bg20

Mir-bg20

Mir-bg21

Mir-bg23

Mir-bg24

Mir-bg24

Mir-bg25
Mir-bg26

Mir-bg26
Mir-bg27

Mir-bg28

Mir-bg29

Mir-bg30

Mir-bg31

Mir-bg32

Mir-bg33b

Mir-bg34a

Mir-bg34b

Mir-bg34c

Mir-bg35

Mir-bg36

Mir-bg37a

Mir-bg37b

Mir-bg37c

Mir-bg38a

Mir-bg38b

Mir-bg38c

Mir-bg39a

Mir-bg39b

Mir-bg40a

Mir-bg40b

Mir-bg40c

Mir-bg41

Mir-bg42

Mir-bg43a

Mir-bg43b

Mir-bg44

Mir-bg45a

Mir-bg45b

Mir-bg46

Mir-bg47

Mir-bg48

Mir-bg49

Mir-bg50

Mir-bg51a

Mir-bg51b

Mir-bg52

Mir-bg53

Mir-bg54

Mir-bg55

Mir-bg56

Mir-bg57

Mir-bg58

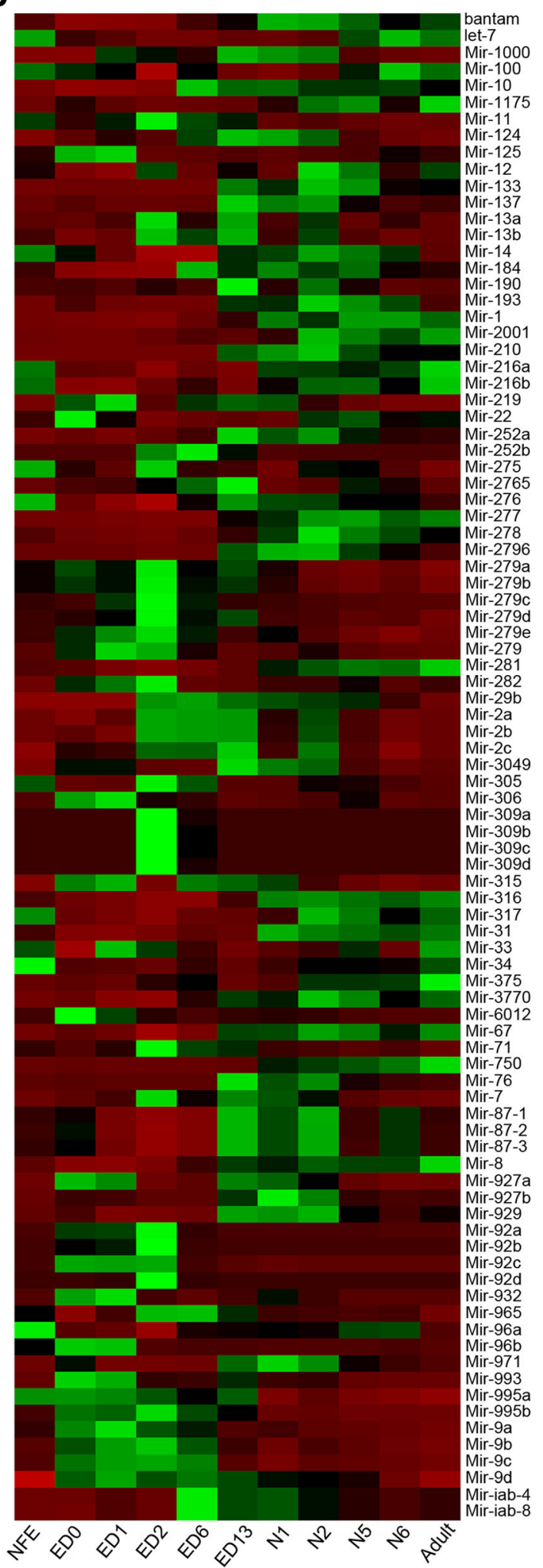

Fig. 2 Expression of B. germanica miRNAs over ontogeny. a Heat map showing the expression of the newly found miRNAs. b Heat map showing the expression of the conserved miRNAs. The abbreviations of the stages are explained in Table 1. c Variation of the expression in conserved and specific miRNAs. The asterisk indicates statistically significant differences between the two samples (Welch's t-test, $p<0.01$ ) 


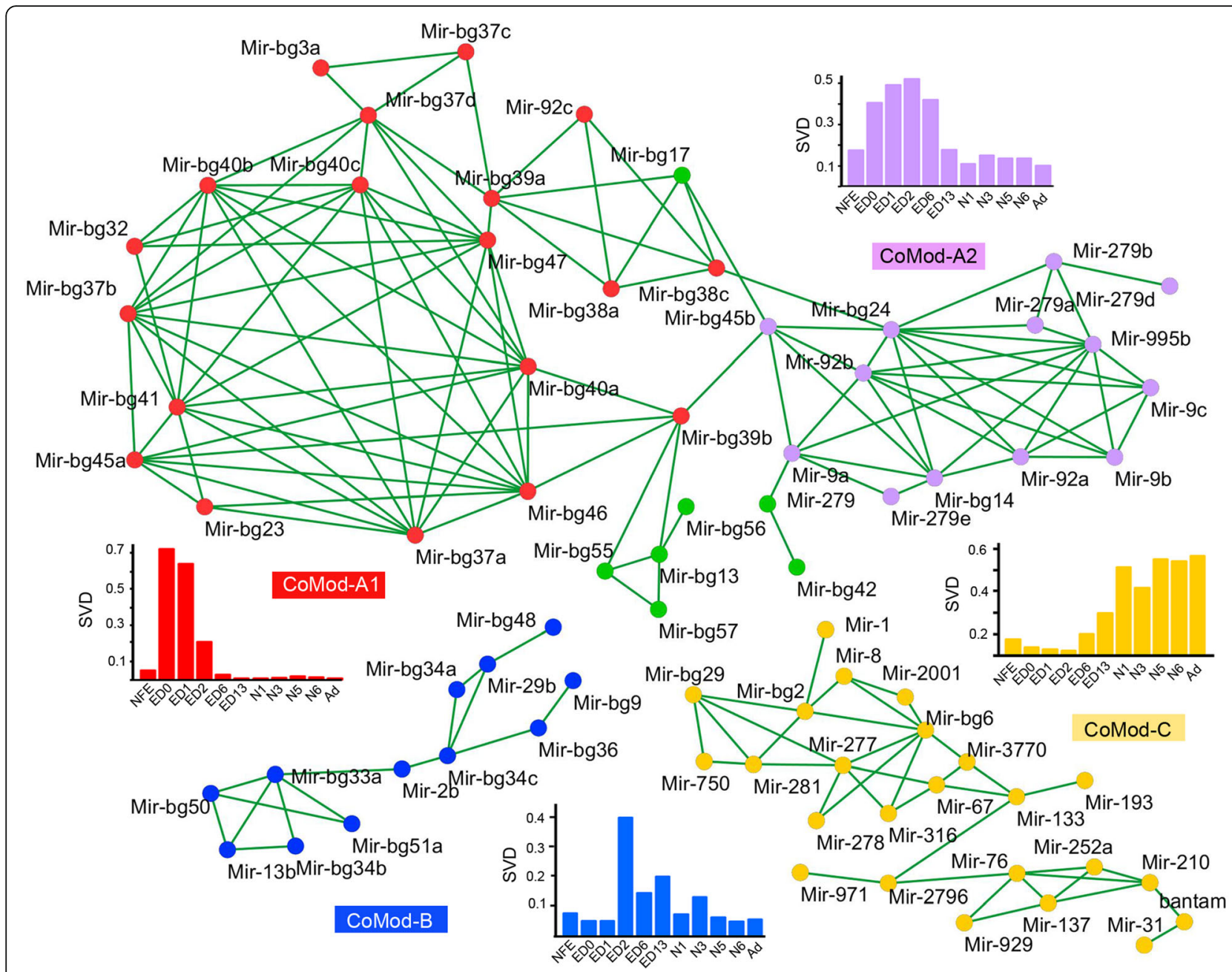

Fig. 3 Coexpression modules of B. germanica miRNAs during ontogenesis. The expression patterns representing each module (metagene expression patterns) are shown besides the corresponding coexpression network

\section{Discussion}

The newly discovered miRNAs predominate in the embryo The present results indicate the embryonic presence of the MIR-309 family of miRNAs in B. germanica, plus 54 novel miRNAs $[10,11]$. D. virilis [16] and T. castaneum [17] have a high percentage of species-specific miRNAs during embryonic life. In the very early embryonic stages of B. germanica, the proportions of miRNAs and piRNAs are similar, resembling those observed in D. melanogaster and D. virilis. T. castaneum, in contrast, shows a high proportion of piRNAs in early ontogenesis [16, 17]. This is likely a peculiarity of the latter species, not attributable to either its germ-band type or mode of metamorphosis.

\section{The four main waves of miRNA expression during cockroach ontogenesis}

The miRNA expression data showed that most miRNA changes occur in the embryo. This is consistent with the hemimetabolan mode of metamorphosis, in which the key developmental process leading to the adult body structure takes place in the embryo. In contrast, the nymphal transitions are developmentally conservative [19]. The transition from N5 to N6 appears to proceed without changes in the miRNAs, which is surprising since adult genetic program should be installed in N6. Also surprising is the small number of changes that occur in the transition from N6 to adult, when the adult program is executed $[15,23]$.

The maternal loading of NFE involves conserved miRNAs such as let-7, Bantam, Mir-34, Mir-305, Mir-8, Mir71 and Mir-1, all of which play roles in basic biological functions [6], as well as large amounts of MIR-bg5 miRNAs. MIR-bg5 is specific for hemimetabolan species [11] and is highly abundant in NFE and in adult females of $B$. germanica. This, plus the observation that the expression of MIR-bg5 miRNAs occur in the ovaries of adult females (see [10], where Mir-bg5 is called "Novel 1"), indicates that 

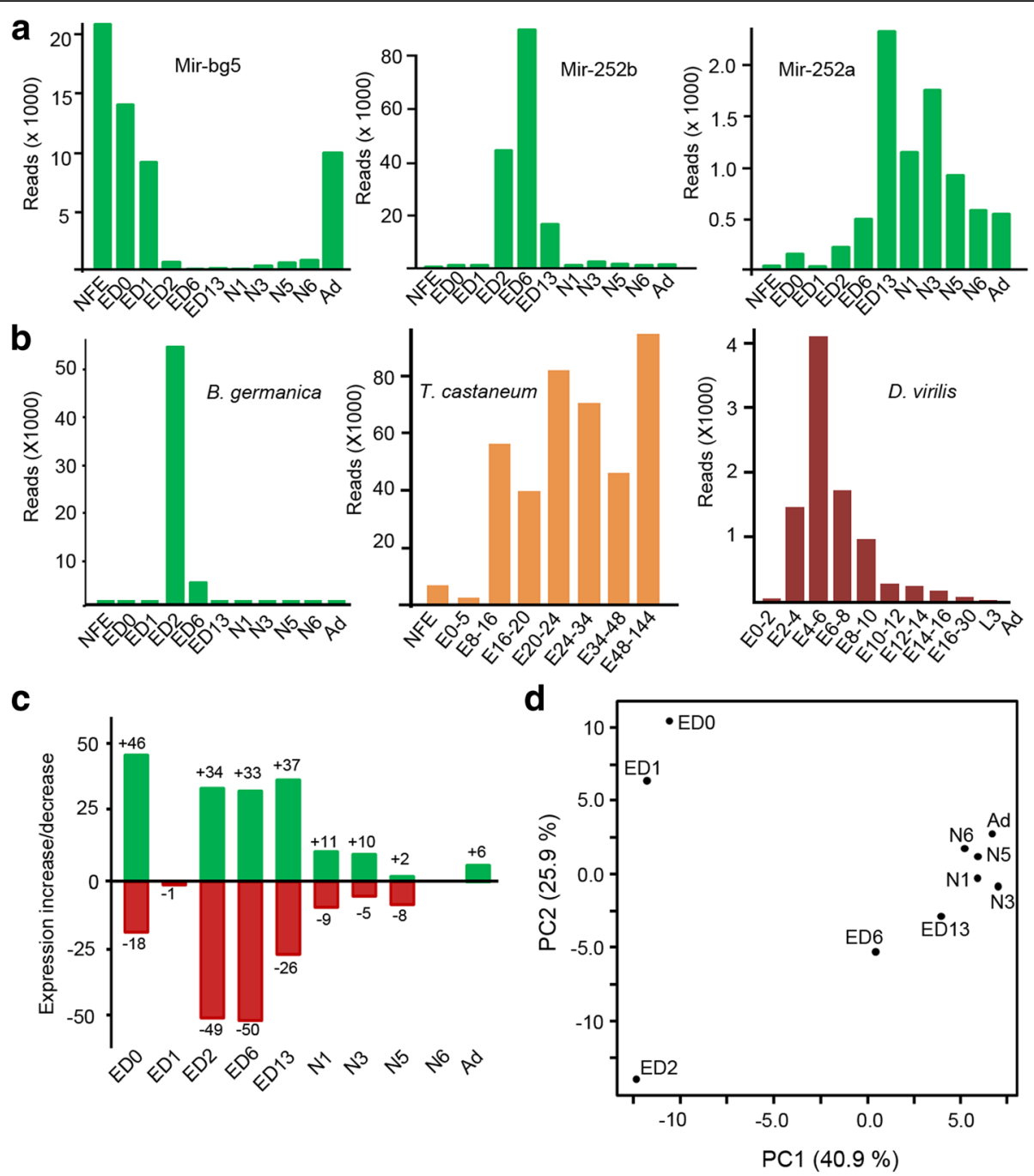

Fig. 4 Changes of miRNAs expression over B. germanica ontogenesis. a Expression of Mir-bg5b, Mir-252b and Mir-252a. b Expression of MIR-309 miRNAs compared to that seen in T. castaneum and D. virilis. $\mathbf{c}$ Number of miRNA genes significantly $(p<0.05)$ upregulated (green) or downregulated (red) at each stage transition. d Principal component analysis plot based on miRNA expression in the embryo, nymph and adult stages. The abbreviations for the stages are explained in Table 1

members of this family are typically maternally loaded miRNAs, at least in B. germanica.

The first two waves of miRNA expression, represented by CoMod-A1 and CoMod-A2, involve Mir-279, which is associated with multiple biological processes [24], and let-7 and Mir-9, which are related with neural differentiation and function [25-27]. A third wave (CoMod-B) that peaks during ED2, contains MIR-2 miRNAs, which are related to cell differentiation, development, morphogenesis and neurogenesis [28]. This wave also involves Mir-29, which is associated to neural cell development [26]. The final wave, represented by CoMod-C, is expressed in late embryos and postembryonic development and involves bantam, Mir-1, Mir-8, Mir-278, Mir- 281, Mir-252 and Mir-31. These
miRNAs are also among the best expressed of larval transcriptomes in D. virilis [16]. The most well-known is bantam, which is associated to basic functions in practically all stages of development [6]. In D. melanogaster larvae, Mir-8 has been related to the regulation of growth factors in body fat [29] and with body size [30], whereas Mir-278 and Mir-277 influence energy homeostasis $[31,32]$. In B. germanica nymphs, Mir-8 regulates atrophin, a factor contributing to neuromotor coordination [14], whereas Mir-252 is involved in the regulation of general growth [13].

\section{The maternal-to-zygotic transition (MZT)}

Degradation and clearance of maternal miRNAs is a key step in the MZT [33, 34], and adenylation at the $3{ }^{\prime}$ end 

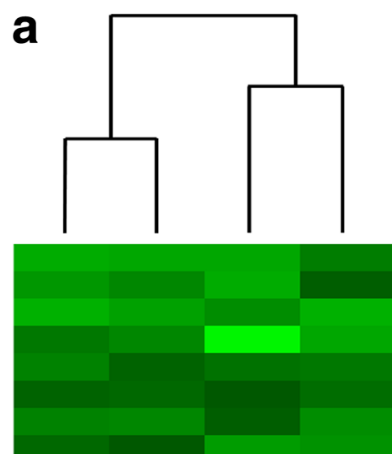

antam

MIR-2

MIR-9

MIR-279

MIR-184

MIR-305

MIR-14

MIR-275

MIR-92

MIR-29

MIR-216

MIR- 6

MIR-31

MIR-10

MIR-276

MIR-315

MIR-750

MIR-1

Let-7

MIR-87

MIR-34

MIR-7

MIR-965

MIR-965

MIR-316

MIR-252

MIR-375

MIR-133

MIR-137

MIR-932

MIR-193

IAB-4

MIR-971

MIR-67

MIR-1000

MIR-210

MIR-76

MIR-929

MIR-124

MIR-22

MIR-1175
MIR-219

MIR-12

MIR-277

MIR-317

MIR-317

MIR-281

MIR-190

MIR-8

MIR-33

MIR-282 b
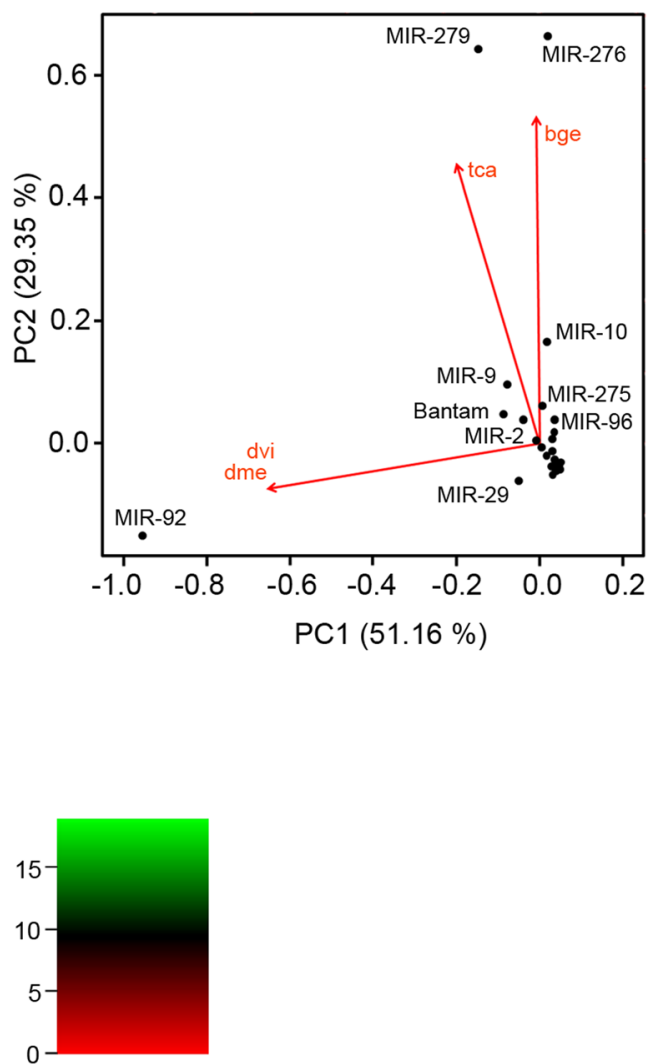

Fig. 5 Families of conserved miRNAs in NFE for B. germanica, T. castaneum, D. melanogaster and D. virilis. a Heat map showing the relative abundance of miRNA families in each species and derived hierarchical clustering. $\mathbf{b}$ Principal component analysis plot showing the main drivers of the clustering. Bge: B. germanica, dme: D. melanogaster, dvi: D. virilis and tca: T. castaneum

of maternal miRNAs has been shown to act as degradation signal. This modification has been observed in the fruit fly, sea urchin and mouse, which indicates that it is evolutionarily conserved [33]. Most miRNAs expressed between NFE and ED1 were highly adenylated at the 3' end. These included the MIR-92 and MIR-10 families, which are also highly adenylated in early embryos of $T$. castaneum [17]. In B. germanica, the proportion of adenylated miRNAs peaked at ED0-ED1 when the relative amount of miRNAs is at its lowest. Adenylation therefore appears to contribute towards the clearing of maternally deposited miRNAs in B. germanica as well.

Although miRNAs are not the only players involved in the degradation of maternal RNAs, they play an important role in this regard. Indeed, our results suggest that a number of early embryo miRNAs might contribute towards clearing maternally loaded mRNAs. In zebrafish, the Mir-430 cluster of miRNAs is key for clearing maternal mRNA in the MZT [35]. In D. melanogaster, maternal mRNA clearing has been associated with the 

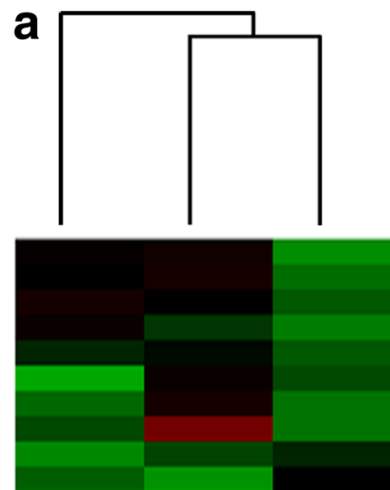
MIR-315 MIR-

MIR-927 MIR-216 MIR-281 MIR-29 MIR-8 MR 7 MIR-7 MIR-305 MIR-31

MIR-12

MIR-317

MIR-965

MIR-316 MIR-34

MIR-375

MIR-375

MR

MIR-87

MIR-87

MIR-750

MR-10

M R-10

MIR-9

MIR-96

MIR-275

MIR-276

MIR-2

Bantam

MIR-184

MIR-92

MIR-279

MIR-124

MIR-282

MIR-137

MIR-76

MIR-33

MIR-1000

ABB-4

MIR-210

MIR-971

MIR-193

MIR-219

MIR-22

MIR-929

MIR-67

MIR-932

b
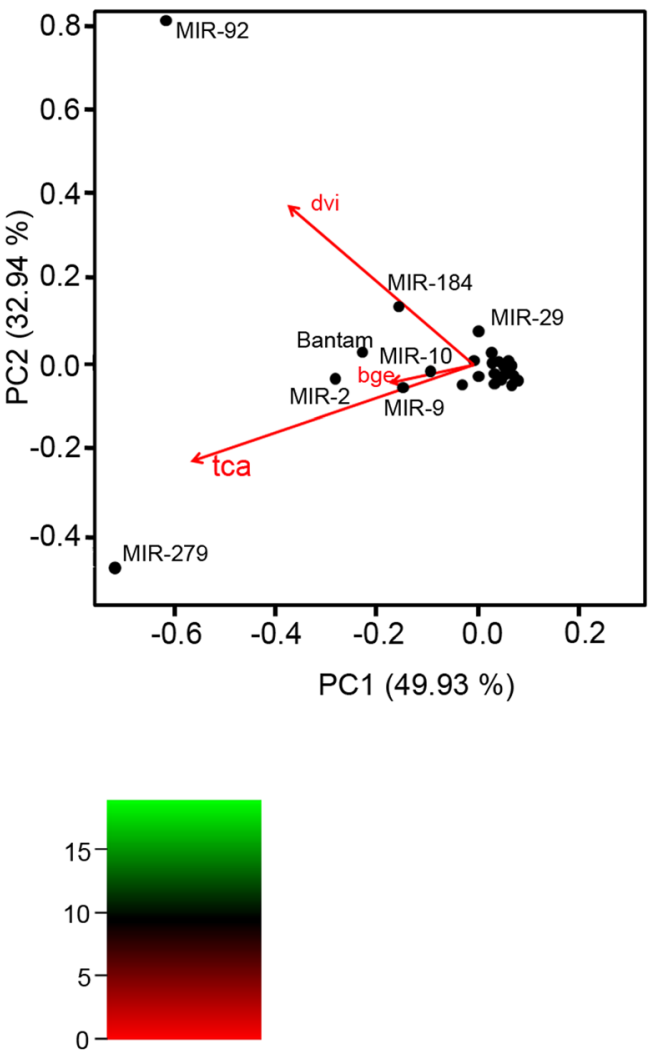

dvi

tca

bge

Fig. 6 Families of conserved miRNAs in EDO for B. germanica, T. castaneum and D. virilis. a Heat map representing the relative abundance of miRNA families in each species and derived hierarchical clustering. b Principal component analysis plot showing the main drivers of the clustering. The libraries were prepared at 1.85\%, 0-3.5\% and 0-6.67\% of development in B. germanica (bge), T. castaneum (tca) and D. virilis (dvi), respectively

Mir-309 cluster, which includes Mir-9/4/79, Mir-5/6/ 2944, Mir-3/309 and Mir-279/286, which are mainly expressed between 9 and $12 \%$ of embryo development [21]. In T. castaneum, these miRNA families also appear involved in regulating maternally deposited mRNAs, along with several additional miRNA families that have been only found in $T$. castaneum, which are expressed between 6 and 11\% of development [17]. In B. germanica, an acute expression peak of the Mir-309 cluster miRNAs (which comprises four Mir-309 paralogs) is observed at ED2 (11\% of development), which might be involved in maternal mRNA clearance. The
miRNAs of the CoMod-B peak at ED2 as well, and might also be involved in maternal mRNA clearance. Finally, Mir-9 and Mir-279, which are associated with maternal mRNA clearance in D. melanogaster [21], belong to CoMod-A2 and show high levels of expression in ED2. Taken together, these data suggest that maternal RNA clearing would involve two successive processes, the first (taking place around ED0-ED1), in which maternal adenylated miRNAs would be degraded, and the second (in ED2), in which maternal mRNAs would be cleared by the activity of Mir-309 and associated scavenger miRNAs. 


\section{miRNAs, germ band and metamorphosis}

Our analyses in the very early stages of embryo development (NFE and ED0) of B. germanica, T. castaneum, D. melanogaster and $D$. virilis indicated that miRNAs from MIR-276 and MIR-279 families are differentially expressed in short germ-band species, whereas those of MIR-92 family are differentially expressed in long germband species. In D. melanogaster, MIR-279 has been related to maternal mRNA clearance in the MZT transition (see above), restricting the emergence of $\mathrm{CO}_{2}$-sensing neurons, the maintenance of circadian rhythm, and the regulation of ovarian border cells [24]. The only reported function for MIR-276 is to promote egg-hatching synchrony by upregulating the transcription coactivator gene brahma in the locust Locusta migratoria [36]. MIR-92 miRNAs downregulate jigr1 in the larval brain of $D$. melanogaster, which is essential for maintaining neuroblast self-renewal [25]. In the tobacco hawk moth Manduca sexta, Mir-92 is clearly overexpressed in embryos compared to larvae, pupae and adults [37]. Mir-92 is expressed in ED0 and ED1 of B. germanica, being one of the miRNAs highly adenylated at the 3 ' end, which suggests that it is cleared in the MZT. If so, then its function could be restricted to very early embryogenesis, possibly to the formation of the germ-band. At NFE and ED0, the let-7 cluster is well expressed in B. germanica and T. castaneum but not in Drosophila spp. The let-7 cluster has traditionally been thought involved in the regulation of developmental transitions [38].

Clustering analyses of B. germanica, T. castaneum and the Drosophila spp. very early embryonic stage libraries did not separate the hemimetabolan and holometabolan species. Differences between these modes of metamorphosis must, however, exist, although they are perhaps clearer in more advanced stages of development, when the body structure is built. MIR-bg5 miRNAs have only been identified in hemimetabolan insects, and have been assumed lost in holometabolans [11, 39]. In B. germanica, they are maternally loaded in high amounts, which indicate them to operate in the early stages of embryo development, thus suggesting that MIR-bg5 miRNAs contribute towards determining the formation of the adult body plan characteristic of hemimetabolan even at the very early stages of embryogenesis.

\section{Conclusions}

The present results show that miRNAs follow welldefined patterns of expression over hemimetabolan ontogeny, patterns that are more diverse during embryonic development than during the nymphal stages. This comes as no surprise since in hemimetabolan species most of the morphogenetic processes are concentrated into the embryonic stages, whereas nymphal development is morphogenetically conservative. The results also suggest that miRNAs play important roles in the developmental transitions between the embryonic stages of development (starting with maternal loading), during which they might influence the maternal-to-zygotic transition, the germ-band type and the metamorphosis mode.

\section{Methods}

Insects

B. germanica specimens were obtained from a colony reared in the dark at $29 \pm 1{ }^{\circ} \mathrm{C}$ and $60-70 \%$ relative humidity. All dissections and tissue sampling procedures were performed on carbon dioxide-anesthetized specimens. Tissues were frozen in liquid nitrogen and stored at $-80{ }^{\circ} \mathrm{C}$ until use.

\section{B. germanica Libraries of small RNAs}

Eleven developmental stages were selected for analysis (Table 1). Data on juvenile hormone $(\mathrm{JH})$ and ecdysteroids (20E) for the chosen stages are from Treiblmayr et al. [40] (JH in nymphal stages), Maestro et al. [41] (JH in embryo stages), Cruz et al. [42] (20E in nymphal stages), Piulachs et al. [43] (20E in embryo stages). Tanaka stages are from Tanaka [44]. For each stage, two small RNA libraries were prepared using the Illumina NEBNext small RNA libraries kit. These libraries were sequenced at the Pompeu Fabra University (Barcelona) Genomic Core Facility using the Illumina NextSeq platform (two runs paired end $\times 50$ cycles).

\section{miRNA nomenclature}

In general, the miRNA nomenclature system proposed by Fromm et al. [20] was followed. For the novel $B$. germanica miRNAs reported here for the first time, the system proposed by Ylla et al. [11] was used; this employs as designator name the code "bg" followed by a number assigned to the novel miRNA.

\section{miRNA predictions}

Low quality reads were filtered out and adapters removed for the 22 small RNA-Seq libraries of B. germanica using Trimmomatic software [45]. Read pairs were then merged using the Pear tool [46]. The resulting reads longer than 18 nts were mapped to the $B$. germanica genome assembly [https://www.hgsc.bcm.edu/arthropods/german-cockroachgenome-project] using Bowtie2 software [47]. The files containing the reads aligned to the genome were used as input for the mirDeep2 miRNA prediction program. Novel miRNA candidates predicted by mirDeep2 [48] were submitted to mirPlot and filtered according to miRNA biogenesis criteria as previously described [11, 20]. Candidates passing all filtering criteria were considered novel miRNAs; these were then grouped into families on the basis of their seed sequence. 


\section{Quantification of miRNA expression}

Small RNA-Seq data for holometabolan insects were obtained from the literature $[16,17]$. These included 10 small RNA-Seq libraries for eight stages of $T$. castaneum embryo development (GSE63770), 21 small RNA-Seq libraries for 12 stages of Drosophila virilis development (GSE54009 \& GSE63770), and two libraries for nonfertilized eggs of D. melanogaster (GSE63770). Four additional libraries for two embryonic stages of $D$. melanogaster were obtained from modEncode [49]. Read adapters were removed using the fastx_clipper algorithm from the fast-toolkit at http://hannonlab.cshl.edu/fastx_toolkit/commandline.htmlt. The read fraction between 16 and 29 nts was then selected. These reads were mapped to the corresponding insect genomes: $T$. castaneum (tca_v4), D. melanogaster (NCBI_build5) and D. virilis (dvir_r1). The number of reads mapping to each annotated miRNA was determined using the FeatureCounts $\mathrm{R}$ package [50]. miRNA annotations for the Drosophila species were retrieved from the miRBase v.21 [51]. For T. castaneum, the miRBase miRNAs were complemented with the 123 miRNAs described by Ninova and collaborators [17]. For B. germanica those previously published were used [11] plus the novel miRNAs described in this paper. Finally, the miRNA counts were normalized as counts per million (CPM) with the edgeR package [52]. For comparing miRNA expression across insects, the miRNA genes were grouped into families as described previously [11]. Only orthologous families present in all compared insects were considered. The expression of each miRNA family was computed as the sum of the CPM corresponding to the miRNA members of that family. 3 '-end modifications of B. germanica miRNAs were further assessed by obtaining those small RNA-Seq reads mapping to an annotated miRNA and containing nucleotides surpassing the 3 '-end boundaries of the miRNA annotation.

\section{Differential expression analysis of miRNAs}

The miRNA expression changes between one stage and the next were determined using the normalization and differential expression functions of the DESeq2 R package [53]. Only those miRNAs with a $p$-value adjusted for a false discovery rate of $<0.05$ were deemed to have a significant change of expression.

\section{Coexpression networks}

The miRNA expression correlation between each pair of miRNA genes was determined using the Spearman correlation coefficient test; this returns the best performance in modeling RNA-Seq coexpression networks [54]. A pair of miRNAs are considered to be connected in the network if their correlation coefficient is higher than 0.9. The largest module was then divided into submodules, applying a correlation coefficient cut-off of 0.925 . The characteristic expression profile of each module and submodule was determined using singular value decomposition [55-57].

\section{Additional files}

Additional file 1: Table S1. Reads obtained from the small RNA libraries sequenced in Blattella germanica. (PDF 18 kb)

Additional file 2: Table S2. Description of the 67 miRNAs newly identified in Blattella germanica. (XLS $41 \mathrm{~kb}$ )

Additional file 3: Table S3. Read counts of each miRNA gene in the Blattella germanica stage-libraries studied. (XLS $56 \mathrm{~kb}$ )

Additional file 4: Figure S1. Coefficient of variation of the expression of newly found and conserved miRNAs during Blattella germanica development. (PDF 10623 kb)

Additional file 5: Figure S2. $P$-values of the differential expression analysis of the miRNA genes at each stage transition during Blattella germanica development. (PDF 4965 kb)

\section{Abbreviations}

CoMod: Co-expression module; CPM: Counts per million; ED: Embryo day (0, 1, 2, 6, 13); kb: Kilobase; miRNA: MicroRNA; MZT: Maternal-to-zygotic transition; N: Nymph (1, 3, 5, 6); NFE: Non-fertilized eggs; nts: Nucleotides; PCA: Principal component analysis; piRNA: Piwi-interacting RNA

\section{Acknowledgments}

Thanks are due to Elena Navas for technical assistance during the preparation of the small RNA libraries. Genomic analyses were performed using the B. germanica genome available at https://www.hgsc.bcm.edu/ arthropods/german-cockroach-genome-project, as provided by the Baylor College of Medicine Human Genome Sequencing Center. We acknowledge support of the publication fee by the CSIC Open Access Publication Support Initiative through its Unit of Information Resources for Research (URICI). G.Y. thanks Hsuan-Cheng Huang and Chen-Ching Lin from the National Yang Ming University of Taipei for providing training in network analysis.

\section{Availability of data and materials}

All the small RNA-Seq data generated have been made publicly available at GEO GSE87031. The GenBank accession number of all miRNA genes newly discovered in B. germanica is indicated in Table $\mathbf{S 2}$.

\section{Authors' contributions}

XB and MDP conceived and supervised the study. GY designed and performed the analyses. All authors discussed and interpreted the results of the analyses. XB prepared the manuscript with comments from MDP and GY. All authors read and approved the final manuscript.

\section{Funding}

This work was supported by the Spanish Ministry of Economy and Competitiveness (grants CGL2012-36251 and CGL2015-64727-P to X.B.), the Spanish Ministry of Science and Innovation (grants BFU2011-22404 and CGL2016-76011-R to M.D.P.) and the Catalan Government (2014 SGR 619). It also received financial assistance from the European Fund for Economic and Regional Development (FEDER funds to X.B. and M.D.P.).

\section{Ethics approval and consent to participate}

This study did not include the use of humans, vertebrate animals of plants directly. No ethics approval is required for experimentation with the study organism, the cockroach Blattella germanica.

\section{Consent for publication}

N/A.

\section{Competing interests}

The authors declare that they have no competing interests. 


\section{Publisher's Note}

Springer Nature remains neutral with regard to jurisdictional claims in published maps and institutional affiliations.

Received: 28 July 2017 Accepted: 5 October 2017

Published online: 11 October 2017

\section{References}

1. Chipman AD. Hexapoda: comparative aspects of early development. In: Evolutionary developmental biology of invertebrates, vol. 5. Vienna: Springer Vienna; 2015. p. 93-110.

2. Peel AD, Chipman AD, Akam M. Arthropod segmentation: beyond the Drosophila paradigm. Nat Rev Genet. 2005;6:905-16.

3. Lynch JA, El-Sherif E, Brown SJ. Comparisons of the embryonic development of Drosophila, Nasonia and Tribolium. Wiley Interdiscip Rev Dev Biol. 2012;1:16-39.

4. Liu PZ, Kaufman TC. Short and long germ segmentation: unanswered questions in the evolution of a developmental mode. Evol Dev. 2005;7:629-46.

5. Bushati N, Cohen SM. microRNA functions. Annu Rev Cell Dev Biol. 2007;23: 175-205.

6. Belles X, Cristino AS, Tanaka ED, Rubio M, Piulachs M-D. Insect MicroRNAs: from molecular mechanisms to biological roles. In: Gilbert LI, editor. Insect molecular biology and biochemistry. Amsterdam: Elsevier-Academic Press; 2012. p. 30-56.

7. Flynt AS, Lai EC. Biological principles of microRNA-mediated regulation: shared themes amid diversity. Nat Rev Genet. 2008:9:831-42.

8. Ebert MS, Sharp PA. Roles for MicroRNAs in conferring robustness to biological processes. Cell. 2012;149:515-24.

9. Hornstein E, Shomron N. Canalization of development by microRNAs. Nat Genet. 2006;38(Suppl):S20-4.

10. Cristino AS, Tanaka ED, Rubio M, Piulachs M-D, Belles X. Deep sequencing of organ- and stage-specific micrornas in the evolutionarily basal insect Blattella germanica (L.) (Dictyoptera, Blattellidae). PLOS One. 2011;6.

11. Ylla G, Fromm B, Piulachs M-D, Belles X. The microRNA toolkit of insects. Sci Rep. 2016;6:37736.

12. Rubio $M$, Belles $X$. Subtle roles of microRNAs let-7, miR-100 and miR-125 on wing morphogenesis in hemimetabolan metamorphosis. J Insect Physiol. 2013;59:1089-94

13. Rubio M, de Horna A, Belles X. MicroRNAs in metamorphic and nonmetamorphic transitions in hemimetabolan insect metamorphosis. BMC Genomics. 2012;13:386

14. Rubio M, Montañez R, Perez L, Milan M, Belles X. Regulation of atrophin by both strands of the mir-8 precursor. Insect Biochem Mol Biol. 2013;43:1009-14.

15. Lozano J, Montañez R, Belles X. MiR-2 family regulates insect metamorphosis by controlling the juvenile hormone signaling pathway. Proc Natl Acad Sci U S A. 2015:112:3740-5.

16. Ninova M, Ronshaugen M, Griffiths-Jones S. Conserved temporal patterns of MicroRNA expression in Drosophila support a developmental hourglass model. Genome Biol Evol. 2014;6:2459-67.

17. Ninova M, Ronshaugen M, Griffiths-Jones S. MicroRNA evolution, expression, and function during short germband development in Tribolium castaneum. Genome Res. 2016;26:85-96.

18. Truman JW, Riddiford LM. The origins of insect metamorphosis. Nature. 1999:401:447-52.

19. Belles X. Origin and evolution of insect metamorphosis. In: eLS. Chichester: John Wiley \& Sons, Ltd; 2011

20. Fromm B, Billipp T, Peck LE, Johansen M, Tarver JE, King BL, et al. A uniform system for the annotation of vertebrate microRNA genes and the evolution of the human microRNAome. Annu Rev Genet. 2015:49:213-42.

21. Bushati N, Stark A, Brennecke J, Cohen SM. Temporal reciprocity of miRNAs and their targets during the maternal-to-zygotic transition in Drosophila. Curr Biol. 2008;18:501-6.

22. Aravin A, Gaidatzis D, Pfeffer S, Lagos-Quintana M, Landgraf P, lovino N, et al. A novel class of small RNAs bind to MILI protein in mouse testes. Nature. 2006;442:203-7.

23. Belles $X$, Santos CG. The MEKRE93 (Methoprene tolerant-Krüppel homolog 1-E93) pathway in the regulation of insect metamorphosis, and the homology of the pupal stage. Insect Biochem Mol Biol. 2014;52:60-8.

24. Sun K, Jee D, de Navas LF, Duan H, Lai EC. Multiple in vivo biological processes are mediated by functionally redundant activities of Drosophila mir-279 and mir-996. PLoS Genet. 2015;11:e1005245.
25. Yuva-Aydemir Y, X-L X, Aydemir O, Gascon E, Sayin S, Zhou W, et al Downregulation of the host gene jigr1 by miR-92 is essential for neuroblast self-renewal in drosophila. PLoS Genet. 2015;11:e1005264.

26. Smirnova L, Gräfe A, Seiler A, Schumacher S, Nitsch R, Wulczyn FG. Regulation of miRNA expression during neural cell specification. Eur J Neurosci. 2005;21:1469-77.

27. Coolen M, Bally-Cuif L. MicroRNAs in brain development and physiology. Curr Opin Neurobiol. 2009;19:461-70.

28. Marco A, Hooks K, Griffiths-Jones S. Evolution and function of the extended miR-2 microRNA family. RNA Biol. 2012;9:242-8.

29. Lee GJ, Jun JW, Hyun S. MicroRNA miR-8 regulates multiple growth factor hormones produced from Drosophila fat cells. Insect Mol Biol. 2015;24:311-8.

30. Jin $\mathrm{H}$, Kim VN, Hyun S. Conserved microRNA miR-8 controls body size in response to steroid signaling in Drosophila. Genes Dev. 2012;26:1427-32.

31. Esslinger SM, Schwalb B, Helfer S, Michalik KM, Witte H, Maier KC, et al. Drosophila miR-277 controls branched-chain amino acid catabolism and affects lifespan. RNA Biol. 2013:10:1042-56.

32. Teleman AA, Maitra S, Cohen SM. Drosophila lacking microRNA miR-278 are defective in energy homeostasis. Genes Dev. 2006:20:417-22.

33. Lee M, Choi Y, Kim K, Jin H, Lim J, Nguyen TA, et al. Adenylation of maternally inherited microRNAs by wispy. Mol Cell. 2014;56:696-707.

34. Marco A. Selection against maternal microRNA target sites in maternal transcripts. G3 (Bethesda). 2015:5:2199-207.

35. Giraldez AJ, Mishima Y, Rihel J, Grocock RJ, Van Dongen S, Inoue K, et al. Zebrafish MiR-430 promotes deadenylation and clearance of maternal mRNAs. Science. 2006;312:75-9.

36. He J, Chen Q, Wei Y, Jiang F, Yang M, Hao S, et al. MicroRNA-276 promotes egg-hatching synchrony by up-regulating brm in locusts. Proc Natl Acad Sci U S A. 2016;113:584-9.

37. Zhang $X$, Zheng $Y$, Jagadeeswaran $G$, Ren $R$, Sunkar R, Jiang $H$. Identification and developmental profiling of conserved and novel microRNAs in Manduca sexta. Insect Biochem Mol Biol. 2012:42:381-95.

38. Faunes F, Larraín J. Conservation in the involvement of heterochronic genes and hormones during developmental transitions. Dev Biol. 2016:416:3-17.

39. Belles X. MicroRNAs and the evolution of insect metamorphosis. Annu Rev Entomol. 2017;62:111-25.

40. Treiblmayr K, Pascual N, Piulachs M-D, Keller T, Belles X. Juvenile hormone titer versus juvenile hormone synthesis in female nymphs and adults of the German cockroach, Blattella germanica. J Insect Sci. 2006;6:1-7.

41. Maestro JL, Pascual N, Treiblmayr K, Lozano J, Belles X. Juvenile hormone and allatostatins in the German cockroach embryo. Insect Biochem Mol Biol. 2010;40:660-5.

42. Cruz J, Martín D, Pascual N, Maestro JL, Piulachs MD, Belles X. Quantity does matter. Juvenile hormone and the onset of vitellogenesis in the German cockroach. Insect Biochem Mol Biol. 2003;33:1219-25.

43. Piulachs M-D, Pagone $V$, Belles X. Key roles of the Broad-Complex gene in insect embryogenesis. Insect Biochem Mol Biol. 2010;40:468-75.

44. Tanaka A. Stages in the embryonic development of the German cockroach, Blattella germanica Linné (Blattaria, Blattellidae). Kontyû, Tokyo. 1976;44:1703-14.

45. Bolger AM, Lohse M, Usadel B. Trimmomatic: a flexible trimmer for Illumina sequence data. Bioinformatics. 2014;30:2114-20.

46. Zhang J, Kobert K, Flouri T, Stamatakis A. PEAR: a fast and accurate Illumina paired-end reAd mergeR. Bioinformatics. 2014;30:614-20.

47. Langmead B, Salzberg SL. Fast gapped-read alignment with bowtie 2. Nat Methods. 2012;9:357-9.

48. Friedländer MR, Mackowiak SD, Li N, Chen W, Rajewsky N. miRDeep2 accurately identifies known and hundreds of novel microRNA genes in seven animal clades. Nucleic Acids Res. 2012;40:37-52.

49. Celniker SE, Dillon LA, Gerstein MB, Gunsalus KC, Henikoff S, Karpen GH, et al. Unlocking the secrets of the genome. Nature. 2009;18:927-30.

50. Liao Y, Smyth GK, Shi W. featureCounts: an efficient general purpose program for assigning sequence reads to genomic features. Bioinformatics. 2014:30:923-30.

51. Kozomara A, Griffiths-Jones S. miRBase: integrating microRNA annotation and deep-sequencing data. Nucleic Acids Res. 2011;39:D152-7.

52. Robinson MD, McCarthy DJ, Smyth GK. edgeR: a bioconductor package for differential expression analysis of digital gene expression data. Bioinformatics. 2010;26:139-40.

53. Love Ml, Huber W, Anders S. Moderated estimation of fold change and dispersion for RNA-seq data with DESeq2. Genome Biol. 2014;15:550. 
54. Ballouz S, Verleyen W, Gillis J. Guidance for RNA-seq co-expression network construction and analysis: safety in numbers. Bioinformatics. 2015;31(13): 2123-30. https://doi.org/10.1093/bioinformatics/btv118.

55. Golub GH, Van LCF. Matrix computations. 3rd ed. Baltimore and London: The Johns Hopkins University Press; 1996.

56. Tomfohr J, Lu J, Kepler TB. Pathway level analysis of gene expression using singular value decomposition. BMC Bioinforma. 2005;6:225.

57. Alter O, Brown PO, Botstein D. Singular value decomposition for genomewide expression data processing and modeling. Proc Natl Acad Sci U S A. 2000;97:10101-6.

Submit your next manuscript to BioMed Central and we will help you at every step:

- We accept pre-submission inquiries

- Our selector tool helps you to find the most relevant journal

- We provide round the clock customer support

- Convenient online submission

- Thorough peer review

- Inclusion in PubMed and all major indexing services

- Maximum visibility for your research

Submit your manuscript at www.biomedcentral.com/submit
Biomed Central 